\title{
Estimating the Probability of Load Curtailment in Power Systems with Responsive Distributed Storage
}

\author{
Ashwin Kashyap \\ Department of Electrical Engineering and Computer Science \\ University of Michigan \\ Ann Arbor, Michigan 48109 \\ Email: ashkash@umich.edu
}

\author{
Duncan Callaway \\ Energy and Resources Group \\ University of California at Berkeley \\ Berkeley, California 94720-3050 \\ Email: dcal@berkeley.edu
}

\begin{abstract}
Controllable electricity loads and storage devices have the potential to significantly and inexpensively increase the operating reliability of power systems, especially in systems with variable, unpredictable generation from renewable sources. However, in cases where a large number of small devices is used for operating reserves, it may be difficult to estimate the available reserve capacity, especially if one desires to minimally impact the end-use function of the devices. To address this issue, we present a probabilistic modeling framework with the specific goal of developing a model of active storage devices, i.e. devices that can both consume and supply electricity to a power system, and to develop bounds on the probability of a load curtailment event based on limited observable characteristics of the devices. The formal analysis in this paper uses asymptotic probability theory to derive bounds for the probability of load curtailment. We expect that this work will form the basis to developing control laws for active device power management as well as for designing reliability-constrained power systems with large active device populations.
\end{abstract}

\section{NOMENCLATURE}

$\Phi(\cdot)$ - Normal cumulative density function

$G$ - conventional generation

$R$ - conventional demand

$T$ - capacity shortage duration

$N$ - number of active devices

$Q_{i}$ - state of charge in the $i^{\text {th }}$ active device

$q_{l}$ - threshold for an active device to be an active load

$q_{u}$ - threshold for an active device to be an active source

$X_{L}$ - number of active loads

$X_{S}$ - number of active sources

$d(\cdot)$ - active load demand as a function of $T$

$L$ - set containing the indices of active loads

$S$ - set containing the indices of active sources

$f(\cdot)$ - probability density function of a continuous random variable

\section{INTRODUCTION}

With advanced metering infrastructure (AMI) becoming commonplace in power systems, it is becoming increasingly feasible to control large (i.e. many thousands), aggregated populations of electricity loads in real time and with limited additional infrastructure investments. One application of such load control is to provide spinning reserve or other ancillary power system services that increase the operating reliability of the bulk system. This could be especially useful in a power system with significant production from renewable generators, such as wind turbines or photovoltaics, whose output is variable and often difficult to predict. Using AMI in this way could provide distribution companies that own the infrastructure with benefits (e.g. self-provision of ancillary services) in addition to remote meter-reading function.

The central concept in this application is that, by reducing electricity consumption - or in the case of distributed storage devices, possibly even returning electricity to the grid - a sudden reduction in output of an electricity generator can be counteracted, and system-wide failure avoided. A request for this type of response could be made on any AMI network capable of broadcasting (for example via multicast addressing) a common signal to all loads. However, the primary purpose of AMI networks is to read interval meter data several times per day; it is unlikely that the network would be built with sufficient capacity to collect state information for all devices on a minute-to-minute basis. As a consequence, it would be impossible to directly measure in real time the capacity of all devices to provide reliability services in aggregate. In this paper we take the alternative route of developing probabilistic models over device states that can be applied to reliability metrics.

The specific objective of this paper is to derive formal bounds on the probability of a load curtailment event (a condition in which total uncontrollable electricity demand exceeds the total capacity of available sources) by estimating the energy capacity of distributed storage devices. To this end, we develop a mathematical framework to describe a power system infrastructure by modeling electricity demand, generation availability, and the behavior of "active devices", which we define to be any agent that can assume the role of an energy source or a load. We assume that it is not possible to query every single active device in real time. Hence, we find a way to work with such uncertainty by using analytical results which require very little information.

We use this model in conjunction with the central limit theorem to develop asymptotic bounds for the probability that a shortfall of generation will lead to load curtailment. Although the model we have developed is relatively general and may find application in other areas, we will focus here on aggregated and controllable energy storage devices as the active device pop- 
ulation. These might include battery backup systems, plug-in hybrid electric vehicles (PHEVs) [6], or perhaps even thermal storage devices (such as water heaters or space conditioning equipment) whose operation can be deferred during periods of generation shortage.

Communications infrastructure other than AMI could be employed (e.g. a residential gateway connected to a broadband interconnection) by third party demand response providers, especially if the distribution companies are unwilling to grant access to their AMI system. The economics of this type of arrangement may be less favorable, especially if small loads are targeted. However, it may be possible to query all devices in real time in this case. We will not address that scenario in this paper. We also note that the method we will describe here would be employed for real-time estimation of the system state, rather than for forecasting.

\section{PREVIOUS WORK}

Reliability theory has been extensively used in the analysis of fault-tolerant systems. A significant number of papers in this line of research has focused on Monte Carlo simulations, both sequential and non-sequential. Billinton et al. [1] have assessed scenarios concerning power system adequacy and have developed simulation models to compute annualized reliability metrics such as loss of load expectation (LOLE). Doherty and O'Malley [2] take a different approach, and develop a model to quantify reserve demand with hourly reliability targets in a power system with significant amounts of uncertainty. For a review of a large portion of the body of work on reliability in power systems, see Allan and Billinton [9] and the references therein.

Relatively little work has been done in the area of evaluating the potential for energy storage devices to contribute to system reliability. Allan et al. [4] studied generation and storage models using sequential Monte Carlo modeling and a network flow algorithm in a constrained optimization framework. Bagen and Billinton [8] use Monte Carlo simulation to determine the impact that energy storage devices have on power system reliability in the presence of intermittent wind turbine generators. Bai et al. [10] look at the energy reserve market consisting of interruptible loads (ILs) and construct a probabilistic framework to illustrate the impact of participants' characteristics on reserve cost allocation. In contrast to the current work, these papers focus on the influence of individual storage devices and assume that, although device states may change unexpectedly, there is no uncertainty in the measured state of the device.

The model introduced in this paper uses random variables to map the physical state of the system to the metric of interest, in this case the probability of load curtailment. This modeling approach has the capacity to capture details at a low level of abstraction, and these details can be aggregated to study the behavior of the whole system.

In contrast to most of the work cited above, which relies on Monte Carlo simulations to quantify reliability metrics, our approach uses probability theory to develop analytical bounds on the probability of load curtailment. Relative to previous research on the specific topic of energy storage device contribution to system adequacy, this work is unique in its reliance on probability theory to establish the aggregate system state. As such, this work requires the presence of a sufficiently large population of devices in the control population to derive analytical bounds. From the purview of the application, it is also a challenge to relate active devices, which are power and energy-constrained, to other sources of generation, which are constrained with respect to power capacity.

\section{The Mathematical Model}

Capital letters have been used to denote random variables. The mean and variance of a random variable are denoted by $\mu$ and $\sigma^{2}$ with appropriate subscripts. Other unknown parameters which characterize distributions are denoted by $\theta$ with appropriate subscripts.

\section{A. Active device distributions and availability}

We quantify the energy in the active device population as follows: Let $N$ denote the number of active devices connected to the grid, which we assume to be known. Let $Q_{i} ; i \in[1, N]$ denote the stored energy, or state of charge, of the $i^{\text {th }}$ active device. We assume that $Q_{i}$ for each device varies according to a stochastic process. For example, in the case of a PHEV, the state of charge is determined by the number of miles traveled by the driver as well as the charging trajectory, both of which are likely to have stochastic components. Although the energy state for any two devices may be the result of different stochastic processes, we assume these processes are not known and that all devices have nominally identical capabilities at the system-level. Therefore we assume the $Q_{i}$ values are independent and identically distributed random variables with finite mean and variance.

Minimally disruptive load control should preserve end-use function to the greatest extent. In this paper, we will assume that active devices with low energy states (or alternatively, whose future power requirements are highest) will not be available to deliver power during a shortfall. We will define availability as follows: First, there is a lower stored energy threshold, $q_{l} \geq 0$ below which an active device has sufficiently low stored energy it must consume power at all times. In this case the device is called an active load. Second, there is an upper energy threshold $q_{u} \geq q_{l}$ above which a device's energy state is sufficiently high that a reduction in stored energy will not significantly impact its end-use; in this case the device is assumed capable of discharging during a shortfall and is called an active source. Note that if $q_{l} \neq q_{u}$, there will be a middle class of neutral devices that can be made to neither consume nor deliver power to the system during the shortfall.

Active loads and sources belong to the sets $L$ and $S$, respectively; these sets have cardinality $X_{L}$ and $X_{S}$. We assume that $X_{L}$ and $X_{S}$ are binomially distributed random variables that can be defined as follows: 


$$
\begin{aligned}
& X_{L} \sim \operatorname{Binomial}\left(N, \theta_{L}\right) \\
& X_{S} \sim \operatorname{Binomial}\left(N, \theta_{S}\right),
\end{aligned}
$$

where $\theta_{L}$ denotes the probability that a randomly chosen device behaves as an active load during a capacity shortage, and $\theta_{S}$ is similarly defined for active sources.

\section{B. Probability of load curtailment}

Our modeling framework is designed to evaluate the probability that the total available generation capacity is insufficient to meet total electricity demand. Specifically, we wish to quantify the probability of load curtailment, or $P L C$, at any given time as follows:

$$
P L C=\operatorname{Pr}(\text { Source capacity }<\text { Load requirement }) .
$$

We define a capacity shortage as an event where uncontrollable demand exceeds available generation. In such situations, active devices may be able to prevent load shedding. Active devices, as defined here, are energy-limited (e.g. energy storage devices and loads that can curtail for a finite period of time before end-use function degrades unacceptably). Therefore, $P L C$ is defined in terms of available energy capacity rather than power, and it is necessary to consider the duration of the capacity shortage.

Let $T$ denote the period during which the active devices will provide reserve capacity. In this paper we will assume $T$ is a fixed value equal to the maximum time that the active devices would be called upon for reserve. In principle, though, $T$ could also be a random variable. We define $G$ to be a discrete random variable (governed by the failure rates of individual generating units, which are assumed to be known) denoting the integral of available generation capacity (i.e. the total energy) over the period $T$. Then the total source energy capacity for the duration is

$$
\text { Source capacity }=G+\sum_{i \in S}\left(Q_{i}-q_{u}\right) .
$$

For simplicity, in this paper the generation model is built with the underlying assumption that all generators have the same power capacity (this assumption could be easily relaxed). Let $k$ be the number of generators and let each be available with probability $\theta_{G}$. Denote the capacity of each generator by $C$. The number of generators working during the period of an energy deficit is a binomial random variable with parameters $\left(k, \theta_{G}\right)$.

Similarly, let $R$ be a continuous random variable, whose distribution is known, denoting total electrical energy consumed by uncontrollable demand (referred to as passive loads throughout the paper) during the same interval $T$, and assume that each active load has power demand $d_{j}, j \in L$. Then the load energy requirement can be written

$$
\text { Load requirement }=R+\left(\sum_{j \in L} d_{j}\right) T \text {. }
$$

We will denote the joint probability distributions governing generation availability and passive load by $f_{G, R}(\cdot, \cdot)$. Although we place no formal restrictions on how these distributions should behave, in general we expect that they will be nonstationary but known a priori. Active device capacities are assumed to be stochastically independent of $G$ and $R$.

We reiterate here that our goal is to determine whether or not the active devices have sufficient energy capacity to prevent a load curtailment. We do not currently model any other detail in the charge-discharge process for the active devices. Specifically, we do not derive active device power management control laws. Rather, for the present study, we assume that a feasible control law exists to distribute the available energy during the generation deficit.

\section{PLC RESUlts}

Combining Equations 1-3, the probability of load curtailment can be written as,

$$
P L C=\operatorname{Pr}\left(\sum_{i \in S} Q_{i}-X_{S} q_{u}+G<R+\left(\sum_{j \in L} d_{j}\right) T\right)
$$

Lemma 1. The total capacity of the active devices can be bounded by the following relation,

$$
\begin{gathered}
\sum_{i \in S \cup L} Q_{i}+\left(N-\left(X_{L}+X_{S}\right)\right) q_{l} \\
\leq \sum_{i=1}^{N} Q_{i} \leq \sum_{i \in S \cup L} Q_{i}+\left(N-\left(X_{L}+X_{S}\right)\right) q_{u}
\end{gathered}
$$

Proof:

$$
\begin{aligned}
\sum_{i=1}^{N} Q_{i} & =\sum_{i \in S \cup L} Q_{i}+\sum_{i \in(S \cup L)^{c}} Q_{i} \\
& \leq \sum_{i \in S \cup L} Q_{i}+\left(N-\left(X_{L}+X_{S}\right)\right) q_{u}
\end{aligned}
$$

Similarly,

$$
\sum_{i \in(S \cup L)^{c}} Q_{i} \geq\left(N-\left(X_{L}+X_{S}\right)\right) q_{l}
$$

Qualitatively, the equations that are in the proof are representative of the fact that neutral devices - those which act as neither active sources nor active loads - have charge capacities that are bounded between $q_{u}$ and $q_{l}$.

Lemma 2. Let $d(T) \triangleq \sup _{j}\left(d_{j}\right)$. Then the probability of load curtailment has the following upper bound,

$$
\left.P L C \leq \operatorname{Pr}\left(\sum_{i=1}^{N} Q_{i}<N q_{u}+R-G+T\left(d(T) X_{L}\right)\right)\right)
$$

Proof: The cumulative density function of a random variable is a monotonically non-decreasing function. Adding 
the capacities of active devices which are not active sources on both sides of the inequality in Equation 4 and using Lemma 1 followed by a direct algebraic simplification gives us a part of the result.

$$
P L C \leq \operatorname{Pr}\left(\sum_{i=1}^{N} Q_{i}<N q_{u}+\left(\sum_{j \in L} d_{j}\right) T+R-G\right)
$$

The second part of the result is obtained by a bound on the cumulative demand of active loads during the period of shortage.

$$
\sum_{j \in L} d_{j} \leq \sum_{j \in L} d(T)=d(T) X_{L}
$$

We now have the setup necessary to invoke the Central limit theorem and the law of large numbers.

\section{Theorem 1.}

$$
P L C \leq \Psi
$$

where,

$\Psi=\int \Phi\left(\frac{N\left(q_{u}-\mu_{Q}\right)+r-g+T\left(d(T) N \theta_{L}\right)}{\sqrt{N} \sigma_{Q}}\right) d f_{G, R}(g, r)$

Proof:

Let $Z$ be a random variable defined as,

$$
Z=\frac{\sum_{i=1}^{N} Q_{i}-N \mu_{Q}}{\sqrt{N} \sigma_{Q}}
$$

From the Central limit theorem, for large values of $N, Z$ converges in distribution to a Gaussian random variable with zero mean and unit variance. Using this property and combining the definition of $Z$ above with Equation 5, we obtain

$$
P L C \leq \operatorname{Pr}\left(Z \leq \frac{N\left(q_{u}-\mu_{Q}\right)+R-G+T\left(d(T) X_{L}\right)}{\sqrt{N} \sigma_{Q}}\right)
$$

We use the law of large numbers on $X_{L}$ as it can be represented as a sum of a large number of Bernoulli random variables. Hence,

$$
X_{L} \approx N \theta_{L}
$$

Using the law of total probability and independence of conventional demand and generation w.r.t active device capacities, together with the distribution of $Z$, we obtain the result.

Figure 1 shows the PLC bounds compared against simulated PLC values as a function of the number of active devices. These simulations use fifty $100 \mathrm{MW}$ generators, each with 0.005 failure probability. The active device SOCs are uniformly distributed from 5 to $20 \mathrm{kWh}$. All active devices are assumed to consume $5 \mathrm{~kW}$ in the absence of a shortfall. The generation
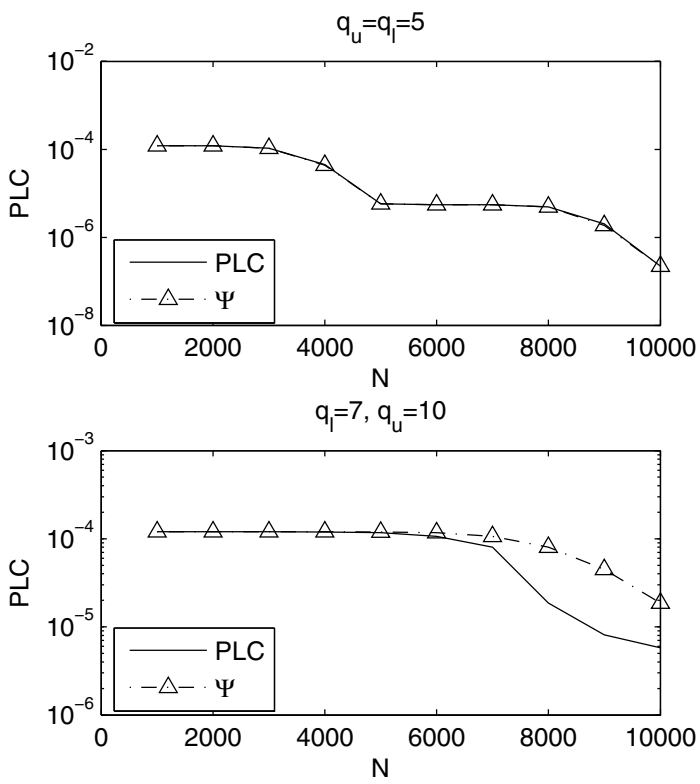

Fig. 1. Upper bound for different $\mathrm{N}$

reserve margin is set to $7 \%$, that is, the sum of available generation is $7 \%$ greater than the sum of all load. We assume that passive load is normally distributed with standard deviation equal to $10 \mathrm{MW}$. For simplicity, we assume that $T$ is always 30 minutes. All the other required parameters were calculated by using the estimation techniques that are described later in the paper.

As the figure shows, increasing the number of active devices providing spinning reserve reduces the PLC, as should be expected. Interestingly, the accuracy of the bounds depends on how close $q_{u}$ is to the smallest value of the support of the SOC distribution. This occurs because, with lower values of $q_{u}$, a greater amount of the SOC distribution is captured in the active device population, thus improving the quality of the CLT approximation.

\section{Vi. Parameter Estimation}

We present a scheme to estimate the unknown parameters in the model described above. This part of the paper hinges on the theory of scalar non-random parametric estimation [7].

\section{A. Active loads, sources and generation}

Since $X_{L}, X_{S}$ and $G$ are all binomially distributed, it is sufficient to present a scheme to estimate the parameters $\theta_{L}, \theta_{S}$ and $\theta_{G}$. We have presented a scheme below to estimate one of these parameters. The same procedure can be repeated to estimate all other parameters as they all belong to the family of binomial distribution with different parameters.

Form a sample set consisting of $n$ independent and identically distributed realizations or measurements from the sample space consisting of active devices and generators.

Let us consider the case of parameter estimation for active loads. We form a set of observations as described above from 
the population of active devices. Each of the measurements are a part of a Bernoulli distribution. Denote the measurements of the unknown parameter for active loads by a vector $x$,

$$
x=\left[x_{1}, x_{2}, x_{3}, \ldots x_{n}\right]
$$

Lemma 3. A complete sufficient statistic for $\theta_{L}\left(\theta_{G}\right.$ or $\left.\theta_{S}\right)$ is given by

$$
T(x)=\sum_{i=1}^{n} x_{i}
$$

Proof:

$$
\begin{aligned}
p_{\theta_{L}}(x) & =\prod_{i=1}^{n} \theta_{L}^{x_{i}}\left(1-\theta_{L}\right)^{1-x_{i}} \\
& =\theta_{L}^{T(x)}\left(1-\theta_{L}\right)^{n-T(x)}
\end{aligned}
$$

By Fisher-Neyman factorization of the probability mass function, we can conclude that $T$ is a sufficient statistic. It can also be shown that the statistic is minimal, and hence is a complete sufficient statistic [7].

A complete sufficient statistic captures the "intrinsic" dimension of the unknown parameter. In our case, it is sufficient to maintain a sum of the observations we make and the number of observations we make to compute the value of $\theta_{L}\left(\theta_{S}\right.$ or $\left.\theta_{G}\right)$.

\section{B. Capacities of active devices}

Since we need to know only the mean and the variance of active device capacities due to the simplification brought about by the Central limit theorem, the following estimators are proposed for $\mu_{Q}$ and $\sigma_{Q}$.

As described in the previous section, form a vector consisting of $n$ i.i.d. realizations of the state of charge and denote the measurements by the vector $x$ as before.

Denote the estimate of $\mu_{Q}$ and $\sigma_{Q}$ by $\hat{\mu_{Q}}$ and $\hat{\sigma_{Q}}$ respectively.

$$
\begin{gathered}
\hat{\mu_{Q}}=\frac{1}{n} \sum_{i=1}^{n} x_{i} \\
\hat{\sigma_{Q}}=\frac{1}{n-1} \sum_{i=1}^{n}\left(x_{i}-\hat{\mu_{Q}}\right)^{2}
\end{gathered}
$$

We use the sample mean and sample variance as estimates of the actual mean and variance of the state of charge in active devices. The flexibility to query a small number of random active devices is very important in implementation of a power management system. Advanced metering infrastructures that are being deployed currently have a wireless communication system to query all the agents in the system periodically.

\section{Accuracy of the estimates}

The CLT approximation requires that we know the first and second moments of the random variable under question. For this purpose, we have proposed a scheme for obtaining estimates of these parameters using a small number of i.i.d. measurements in the sample space. A fundamental question regarding the accuracy of these estimates can be answered by using concentration inequalities. Specifically, we use Hoeffding's inequality to establish a relation between the number of measurements and the accuracy of the estimate.

We have shown earlier that a sufficient statistic for estimation of the unknown parameters is the sum of the observations. We use the fact that the measurements are i.i.d. and also that the sample mean is an unbiased estimate of the actual mean. A formal statement regarding the above mentioned points can be seen below.

Lemma 4. Let $X_{1}, X_{2}, \ldots, X_{n}$ be the random variables denoting the measurements of the state of charge from the sampling population. The state of charge is assumed to have a finite support in the interval $\left[q_{\min }, q_{\max }\right]$. Let

$$
Y=X_{1}+X_{2}+\ldots+X_{n}
$$

Hoeffding's inequality states that,

$$
\operatorname{Pr}(|Y-E(Y)| \geq n t) \leq \exp \left(-\frac{2 n^{2} t^{2}}{\sum_{i=1}^{n}\left(q_{\max }-q_{\min }\right)^{2}}\right)
$$

It is easy to see that $E(Y)=n \mu_{Q}$ and that $\frac{Y}{n}$ is an unbiased estimator for the mean of the state of charge distribution. Rearranging the terms and simplifying Hoeffding's inequality, we get,

$$
\operatorname{Pr}\left(\left|\frac{Y}{n}-\mu_{Q}\right| \geq t\right) \leq \exp \left(-\frac{2 n t^{2}}{\left(q_{\max }-q_{\min }\right)^{2}}\right)
$$

The right hand side of the inequality shows that the variation of the sample mean from the actual mean dies down exponentially with an increase in the number of measurements. It is possible to fix the variation by taking an appropriate number of measurements. This forms the basis of the $(t, \delta)$-approximation algorithm where we randomly sample from the population and output the parameter which varies at most by $t$ from the actual parameter value. This approximation guarantee holds with a probability of $1-\delta$, where $\delta$ denotes the expression on the right hand side of the inequality.

\section{Example}

We use the inequality described above to illustrate the order of magnitude of the sampling size. Let us assume that the active device population consists exclusively of PHEVs with a battery capacity of $10 \mathrm{kWh}$. Further, let us assume that the minimum state of charge that can be attained is arbitrarily close to zero. This translates to $q_{\min }=0$ and $q_{\max }=10 \mathrm{kWh}$. If we fix the accuracy of our estimate to within $0.5 \mathrm{kWh}$ of the actual parameter value with a probability of 0.9 , the number 


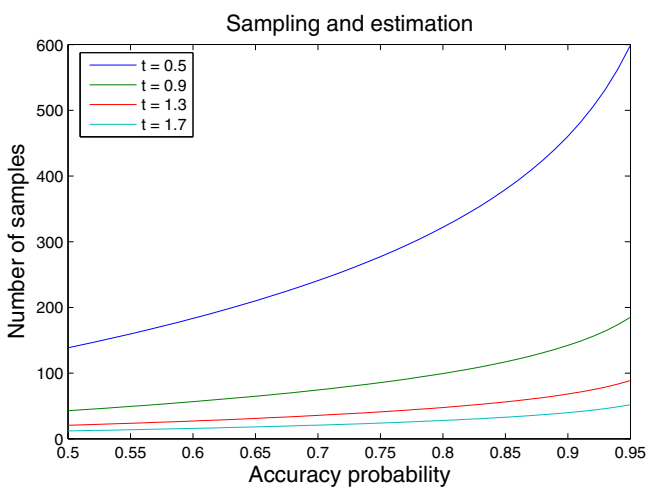

Fig. 2. Accuracy of estimated parameters

of samples to be taken from the population is approximately 470. Figure 2 has a set of curves to depict the variation in the sample size with accuracy probability for different levels of accuracy of the estimates.

\section{CONCLUSIONS AND FUTURE WORK}

A mathematical model describing the prominent agents in a power system has been developed. The concept of estimating the ability of active storage devices whose state is uncertain to support system reliability during periods of generation shortage has been explored. We have presented rigorous results for the probability of load curtailment using the Central limit theorem. The analytical approach we take here is intended to complement the existing Monte-Carlo simulation schemes in the literature of power systems and control theory.

In future work, we plan to develop efficient centralized and distributed control algorithms to distribute the available active device capacity during an energy deficit. With this, we intend to consider the charging and discharging profiles of the active device population, which impose physical constraints on how the available energy can be distributed. By considering the physical limitations of AMIs in sampling and gathering information about all the devices connected to the grid, we will develop a computationally efficient information management scheme to handle the device population during "discharge-togrid" events.

\section{ACKNOWLEDGEMENTS}

This work was supported by the National Science Foundation Grant No. 0835995 and the California Institute for Energy and Environment.

\section{REFERENCES}

[1] Billinton, R. and Wangdee, W., "Impact of utilising sequential and nonsequential simulation techniques in bulk-electric-system reliability assessment", IEE-Proceedings - Generation, Transmission and Distribution, 2005, pp 623-628

[2] Doherty, R. and O'Malley, M., "A new approach to quantify reserve demand in systems with significant installed wind capacity", IEEE Transactions on Power Systems, 2005, pp 587-595

[3] Malhame, R. and Chee-Yee Chong, "Electric Load Model Synthesis by Diffusion Approximation of a High-Order Hybrid-State Stochastic System", IEEE Transactions on Automatic Control, 1985, pp 854-860
[4] Allan, R.N., Li, R., Elkateb, M.M., "Modelling of pumped-storage generation in sequential Monte Carlo production simulation", IEEProceedings - Generation, Transmission and Distribution, 1998, pp 611615

[5] Edward P. Kahn, "Effective Load Carrying Capability of Wind Generation: Initial Results with Public Data", The Electricity Journal, Volume 17, Issue 10, December 2004, pp 85-95

[6] Willett Kempton and Jasna Tomic, "Vehicle-to-grid power implementation: From stabilizing the grid to supporting large-scale renewable energy", Journal of Power Sources, 2004

[7] Vincent Poor, "An Introduction to Signal Detection and Estimation", Springer, 1998

[8] Bagen and Roy Billinton, "Impacts of energy storage on power system reliability performance", Canadian Conference on Electrical and Computer Engineering, 2005

[9] Billington, R. and Allan, R. N., "Reliability evaluation of power systems", 2nd ed. New York: Plenum Press.,, 1996

[10] Bai, J.; Gooi, H.B.; Xia, L.M.; Strbac, G.; Venkatesh, B, "A probabilistic reserve market incorporating interruptible load", IEEE Transactions on Power Systems, 2006, pp 1079-1087 